\title{
A multidisciplinary consensus on the morphological and functional responses to immunotherapy treatment
}

\author{
L. Leon-Mateos ${ }^{1}$ - M. J. Garcia-Velloso 2,3 ${ }^{\text {(1) }} \cdot$ R. García-Figueiras ${ }^{4} \cdot$ J. F. Rodriguez-Moreno ${ }^{5}$ J. L. Vercher-Conejero ${ }^{6}$ \\ M. Sánchez ${ }^{7} \cdot$ J. L. Perez Gracia ${ }^{8} \cdot$ M. Simo-Perdigo ${ }^{9} \cdot$ L. Gorospe $^{10}$
}

Received: 25 January 2020 / Accepted: 22 June 2020 / Published online: 4 July 2020

(c) The Author(s) 2020

\begin{abstract}
The implementation of immunotherapy has radically changed the treatment of oncological patients. Currently, immunotherapy is indicated in the treatment of patients with head and neck tumors, melanoma, lung cancer, bladder tumors, colon cancer, cervical cancer, breast cancer, Merkel cell carcinoma, liver cancer, leukemia and lymphomas. However, its efficacy is restricted to a limited number of cases. The challenge is, therefore, to identify which subset of patients would benefit from immunotherapy. To this end, the establishment of immunotherapy response criteria and predictive and prognostic biomarkers is of paramount interest. In this report, a group of experts of the Spanish Society of Medical Oncology (SEOM), the Spanish Society of Medical Radiology (SERAM), and Spanish Society of Nuclear Medicine and Molecular Imaging (SEMNIM) provide an up-to-date review and a consensus guide on these issues.
\end{abstract}

Keywords Immunotherapy $\cdot$ Response $\cdot$ Imaging $\cdot$ Biomarker $\cdot$ Immune-related adverse events

\section{Immunotherapy clinical application}

The implementation of immunotherapy has radically changed the treatment of oncological patients. Unlike other oncological treatments (such as chemotherapy, tyrosine kinase inhibitors and others that act directly upon tumor cells), immunotherapy exerts antitumor action by activating the patient's immunologic response against cancer and

L. Leon-Mateos, M. J. Garcia-Velloso and R. García-Figueiras authors have coordinated this manuscript and have contributed equally to its preparation.

M. J. Garcia-Velloso

mjgarciave@unav.es

1 Medical Oncology Department, Instituto de Investigación Sanitaria de Santiago de Compostela (IDIS),

Complexo Hospitalario Universitario de Santiago de Compostela (CHUS), Servicio Galego de Saude, Santiago de Compostela, Spain

2 Nuclear Medicine Department, Clinica Universidad de Navarra, Av. Pio XII 36, 31008 Pamplona, Spain

3 IdisNA, Instituto de Investigación Sanitaria de Navarra, Pamplona, Spain

4 Radiology Department, Hospital Clínico Universitario de Santiago de Compostela, Santiago de Compostela, Spain can be used as a monotherapy or in combination with other immunotherapeutic agents, chemotherapy or targeted therapies [1-6]. Currently, immunotherapy is approved or under development for the treatment of patients with head and neck tumors, melanoma, lung cancer, bladder tumors, colon cancer, cervical cancer, breast cancer, Merkel cell carcinoma, liver cancer, leukemia and lymphomas (Fig. 1). However, its efficacy is restricted to a limited number of cases. The challenge is, therefore, to identify which subset of patients would benefit from immunotherapy [7]. There are also other important areas of research, such as the establishment of

5 Medical Oncology Department, Centro Integral Oncologico HM Clara Campal, Madrid, Spain

6 Nuclear Medicine Department - PET Unit, Bellvitge University Hospital - IDIBELL, Barcelona, Spain

7 Radiology Department, CDIC, Hospital Clínic, Barcelona, Spain

8 Oncology Department, Clinica Universidad de Navarra, Pamplona, Spain

9 Nuclear Medicine Department, Hospital Universitari Vall Hebron, Barcelona, Spain

10 Radiology Department, Ramón y Cajal University Hospital, Madrid, Spain 


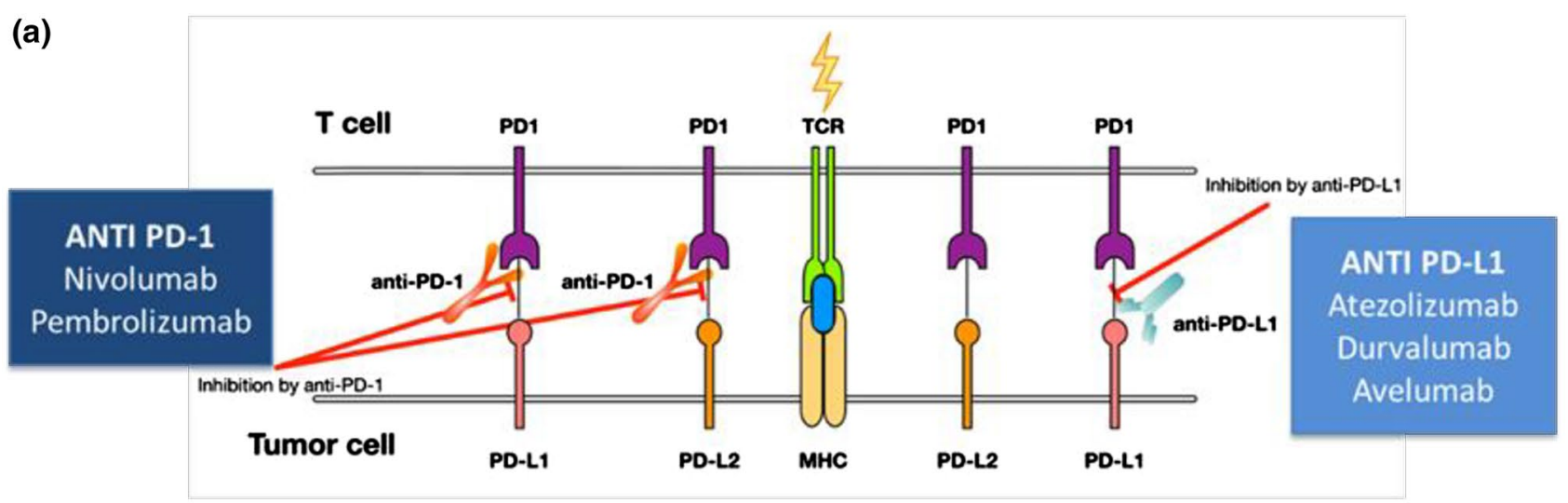

(b)

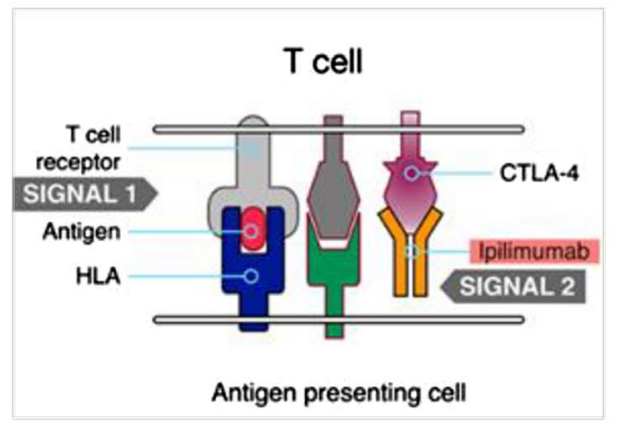

Fig. 1 Mechanism of action of immunotherapy and main general indications. a The $\mathrm{T}$ lymphocyte recognizes the antigen presented by the major histocompatibility complex (MHC) of the tumor cell. The ligands PD-L1 and PD-L2 function as negative regulators of the immune response by binding to the lymphocyte receptor PD-1. The anti-PD-1 and anti-PD-L1 antibodies interfere with this bind-

treatment response criteria according to drug mechanisms of action and identifying and managing immune-mediated toxicities.

A group of experts from the Spanish Society of Medical Oncology (SEOM), the Spanish Society of Medical Radiology (SERAM), and Spanish Society of Nuclear Medicine and Molecular Imaging (SEMNIM), selected and supported by these scientific societies, met to discuss and provide an up-to-date introductory review on some general issues on immunotherapy, and a multidisciplinary consensus on the immunotherapy response criteria and the predictive and prognostic biomarkers. In addition, functional and molecular imaging advances for immunotherapy response assessment are also reviewed. The consensus was initiated with a faceto-face meeting, where the content, procedures and topic

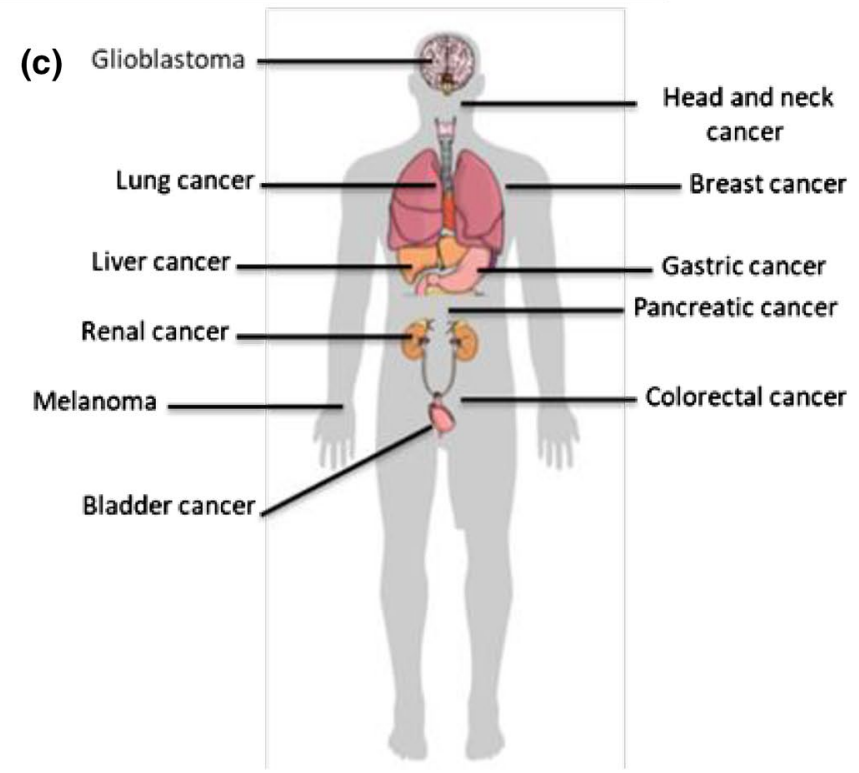

ing, potentiating the immune response against the tumor. b CTLA-4 is another receptor that inhibits the action of $\mathrm{T}$ lymphocytes. The monoclonal antibody ipilimumab blocks CTLA- 4 and potentiates the activity of T lymphocytes. $\mathbf{c}$ Several modalities of immunotherapy are approved or in development for use in different tumor locations

distribution among the experts were made. The first draft of the consensus was distributed among all participants who made comments and amendments to the document that were discussed via e-mail and teleconference. The coordinators of the consensus agreed a second draft based on the comments received. This process was repeated twice until a final draft was circulated, agreed and approved by all authors.

\section{Immunotherapy types and mechanisms of action}

Immunotherapy is a general term comprising several strategies for triggering the host immune response, including the following: 

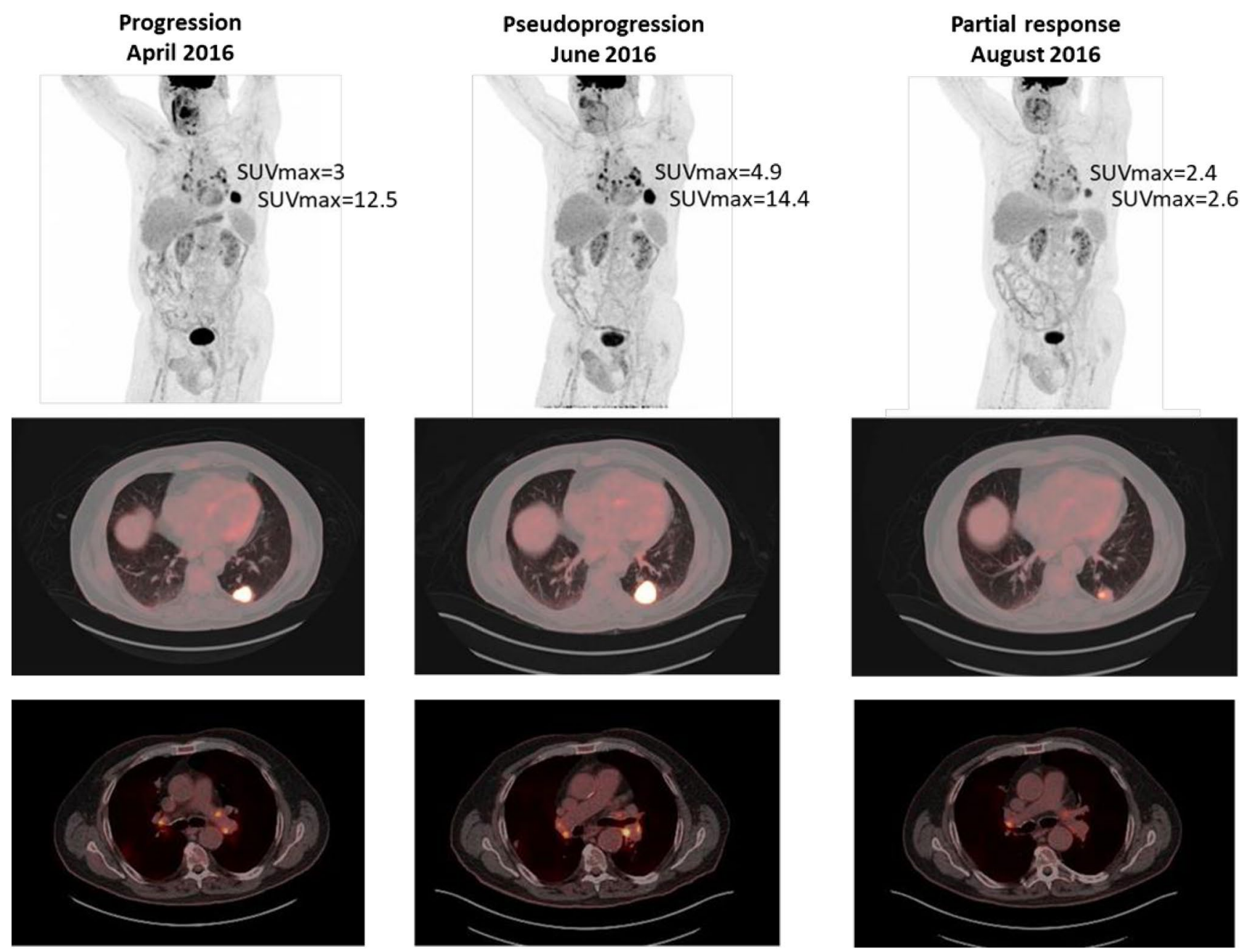

Fig. 2 Pesudoprogression in a patient with lung adenocarcinoma. Patient diagnosed in January 2014 of well-differentiated lung adenocarcinoma T4N3M0 stage, and treated with cisplatin and pemetrexed. Progression resistant to a second-line chemotherapy was detected in April 2016. ${ }^{18} \mathrm{~F}$-FDG PET/CT (a) identified a lesion located in LII (SUVmax of 12.5) and mediastinal and hilar lymph node uptake (SUVmax of 3). Nivolumab was started and an ${ }^{18}$ F-FDG PET/ CT study 2016 was performed for response assessment in June (b)

1. Checkpoint inhibitors. The immune response involves complex signal balances between $\mathrm{T}$ lymphocyte cytotoxic activation and inhibition [8]. Checkpoint inhibitors induce a positive balance that favors the activation of effector T lymphocytes.

2. Cytokines. Cytokines (interferon $\alpha / \beta$, interleukin 12 , etc.) facilitate adequate lymphocyte activation. Although their clinical use is limited by their marked toxicity, some cytokines, such as interleukin 2 or alpha $2 b$ interferon, have shown results for treating melanoma and renal cell carcinoma [9].

3. Innate immunity activation. Nonspecific initial immune response activation might also facilitate cancer progression management. One example could be adjuvant revealing an increase in the metabolism and size of the lung lesion (SUVmax of 14.4) and in the number and metabolism of thoracic lymph nodes (SUVmax of 4.9). Because of clinical stability, immunotherapy was maintained and ${ }^{18} \mathrm{~F}-\mathrm{FDG}$ PET/CT performed in August 2016 (c) showed a metabolic reduction of lung lesion (SUVmax of 2.4) and lymph nodes (SUVmax of 2.6), consistent with partial response. Given the evolution, the ${ }^{18} \mathrm{~F}$-FDG PET/CT(B) was interpreted as pseudoprogression

treatment of urothelial carcinoma with Calmette-Guérin bacillus (BCG) [10].

4. Cell therapy. Different strategies consist of manipulating patient cells to restore their capacity to recognize and kill tumor cells.

4.1 Tumor-Infiltrating Lymphocytes (TILs): TIL isolates are taken from tumor biopsies and replicated in ex vivo cell culture for their later infusion in patients previously submitted to myeloablative treatments [11].

4.2 Chimeric antigen receptor (CAR) - T cells: patient's $\mathrm{T}$ lymphocytes are extracted by leukapheresis and genetically modified ex vivo to recognize a given 
preselected antigen. Such therapy has shown remarkable activity against hematological neoplasms [12].

5. Oncolytic viruses. human viruses have been genetically modified to infect and kill tumor cells, creating a proinflammatory microenvironment triggering additional adaptive immune mechanisms [13].

6. Vaccines. specific antitumor response activation can be elicited by active immunization (vaccine administration) derived from cancer cell expressions of certain tumorspecific antigens.

\section{Unusual response patterns associated with immunotherapy}

Tumor response to oncologic treatments has been associated with tumor size reduction. However, immunotherapy may usually cause several atypical response patterns, such pseudoprogression, hyperprogression, and dissociated responses, that are difficult to evaluate according to classic tumor response criteria and with conventional imaging techniques. Besides, immunotherapy may also achieve durable responses in a subset of patients with advanced cancer that can be maintained even after stopping treatment.

\section{Sustained responses}

Sustained responses are one of the main response patterns associated with immunotherapy. A major breakthrough of immunotherapy is its potential to achieve lasting responses in a subset of patients with advanced cancer that can be maintained even after stopping treatment. There is no standard definition of sustained response in the literature. This kind of response appears in all cancer types, and there are current investigations underway to identify predictive factors of this feature [14-16].

\section{Pseudoprogression}

Tumor shrinkage has been identified after an initial increase in tumor burden or the appearance of new lesions. This phenomenon, called pseudoprogression, is the consequence of treatment-activated immune cells infiltrating the tumor milieu. It is associated with edema or necrosis that may cause a radiographic increase in tumor volume, including the appearance of lesions not recognized on previous imaging $[17,18]$. Pseudoprogression is the most characteristic atypical response pattern associated with immunotherapy. It was first reported in melanoma patients treated with antiCTLA4, but this response pattern may also appear with other immunotherapy agents and in different tumor types
(Fig. 2) [19-21]. Pseudoprogression can appear early during treatment (within the first 12 weeks of therapy) but can also be a late phenomenon. A recent study in melanoma patients showed an early pseudoprogression rate of $4.6 \%$ and a late pseudoprogression rate of $2.8 \%$ [20]. There is a variable pseudoprogression rate among different tumor types. The rate is higher in the case of melanoma (up to $10 \%$ of patients) [22] and lower in epithelial malignancies such as lung cancer and urothelial carcinoma (1.5-3.0\%). In this setting, we should consider that most of the cases of possible pseudoprogression reflect real progression. However, there is a need to establish specific response criteria for immunotherapy allowing for the continuation of treatment after apparent progression, according to classic criteria in clinically fit patients without associated toxicity.

\section{Hyperprogression}

Hyperprogression is defined as tumor growth after the initiation of immunotherapy that is faster than under previous treatment and has been described based on retrospective observations [23]. The rate of hyperprogression is variable, ranging from 4 to $29 \%$ for different agents and tumor types [24]. Hyperprogression is prone to controversies and shows considerable incidence variability due to clinical trial heterogeneity. It is difficult to establish a difference between hyperprogression as an independent entity and rapid progression already observed in oncologic patients. Different papers published have defined multiple parameters to assess and define hyperprogression, such as the "tumor growth rate (TGR)" and the "time-to-treatment failure (TTF)". These parameters usually combine Response Evaluation Criteria in Solid Tumors (RECIST) and elapsed time between assessments [25].

Hyperprogression correlates with worse patient survival rates. Several studies have been conducted to identify hyperprogression risk factors, including advanced age, multiple metastatic lesions, and previous radiotherapy [23, 26, 27].

\section{Dissociated response}

A dissociated response is considered when there is regression in some target lesions but progression in others. In this scenario, it would be important to identify patients with oligoprogression, due to the possibility of treating these lesions locally. This pattern has already been described in the context of conventional chemotherapy and especially in the case of targeted therapies. A study including lung cancer patients treated with immunotherapy described a $7 \%$ rate of dissociated response [21]. In this study, patients with pseudoprogression or dissociated response showed better survival. 


\section{Key points and recommendations}

Novel patterns of response and progression to immunotherapy, which may be more frequent in immunotherapy than in chemotherapy or targeted therapies, should be known.

\section{Immune-related adverse events}

Apart from these atypical response patterns, immunerelated toxicities may appear during treatment due to selfimmunity deregulation or induction. The most common adverse event is dermatologic toxicity, but immune-related toxicity could affect any organ and cause different clinical and radiological manifestations that might mimic tumor progression (Table 1) [28]. These adverse events must be identified to initiate immunosuppression treatment. Pneumonitis is the most common thoracic complication, with different radiologic manifestations [29]. A nodular pattern of organized pneumonia or sarcoidosis/sarcoid reaction could be confounded with disease progression. To differentiate these adverse events from progression, it is important to ascertain whether there is only thoracic progression and to compare the initial tumor radiological characteristics with the current tumor manifestations [30]. Other toxicities with doubtful radiologic or metabolic manifestations that could be confounded with progression are pituitary gland disorders, thyroiditis, pericarditis, colitis and pancreatitis, all characterized by volume augmentation of the different organs and increased [18F]fluorodeoxyglucose $\left({ }^{18} \mathrm{~F}-\mathrm{FDG}\right)$ uptake in positron emission tomography (PET) [31, 32].

Table 1 Main immune-related adverse events in patients treated with immunotherapy that could interfere with the response assessment

$\begin{array}{ll}\text { Pulmonary } & \text { Pneumonitis } \\ \text { Gastrointestinal } & \text { Sarcoidosis/sarcoid reaction } \\ & \text { Colitis } \\ & \text { Hepatitis } \\ \text { Pancreatitis } \\ \text { Primary hypothyroidism } \\ \text { Hyperthyroidism } \\ \text { Primary adrenal insufficiency } \\ \text { Hypophysitis } \\ \text { Cardiovascular } & \text { Myocarditis } \\ & \text { Pericarditis } \\ & \text { Vasculitis } \\ & \text { Venous thromboembolism }\end{array}$

\section{Key points and recommendations}

- The main clinical and imaging manifestations of immune-related toxicities must be known to make an early diagnosis and to initiate early treatment of these adverse events.

- FDG-PET is a non-invasive imaging technique that allows precise localization of all potential immunerelated adverse effects and to monitor the response to immunosuppression treatment.

\section{Immunotherapy response criteria}

Tumor therapy response is categorized into four main groups: complete response [CR], partial response [PR], progressive disease [PD], and stable disease [SD]. During the last decades, several radiologic and metabolic response criteria for different oncological treatments have been described [33]. The appearance of new therapeutic strategies in recent years (targeted therapies, antiangiogenic agents and, more specifically, immunotherapy) has revealed some limitations of these criteria, such as the possibility of not detecting atypical response patterns (such as pseudoprogression) [34]. In this section, we will review the different imaging criteria described for the assessment of immunotherapy response.

\section{Anatomic response criteria}

All radiologic response criteria for immunotherapy share common aspects, such as being based on target lesion dimension changes (measurable lesions, most representative of total tumor burden) and nontarget lesions (all the other measurable and unmeasurable lesions) defined in baseline studies. In short, differences among several response criteria to assess immunotherapy treatment are based on measurement methods (single, if based on RECIST criteria; or bidimensional, if based on the World Health Organization (WHO) measurements) or on the consideration of new lesions (specifically unmeasurable lesions) to define if there is progression or not; the criteria also differ in regard to incorporating new lesions in the baseline tumor burden (Table 2).

The anatomic or radiologic immunotherapy response criteria include the following:

- RECIST 1.1: Version 1.1 of the "conventional" RECIST criteria introduced in 2009 are the most frequently used criteria for solid tumor treatment response assessment. These criteria are the only criteria that have been validated and accepted by the main regulatory agencies [3538]. However, according to RECIST, increased tumor 


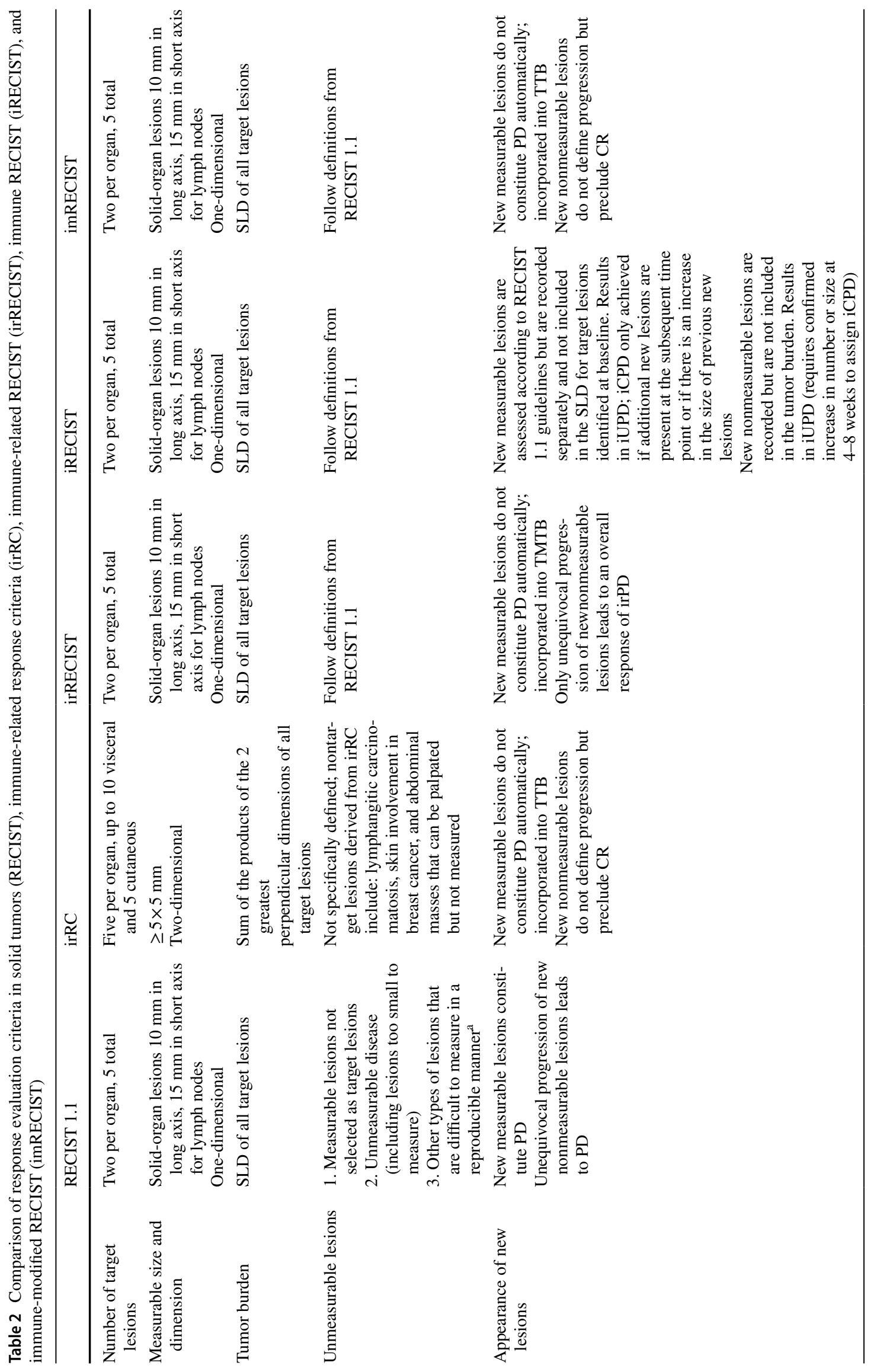







size or the appearance of new lesions in a cancer patient would invariably imply PD, which would result in an incorrect assessment of those patients with pseudoprogression, for instance.

- Immune-related response criteria (irRC): The irRC were introduced in 2008, and their main contribution was to incorporate new measurable lesions into baseline reference index lesions according to a new concept called "total tumor burden". In addition, unlike the timeline of the classic criteria, disease progression should be confirmed within 4 weeks. Some limitations of the irRC are as follows: (1) Based on the WHO criteria, the irRC cannot be compared to the RECIST criteria because of the use of bidimensional-type measurements; (2) worse assessment of node involvement; and (3) possible overestimation of treatment efficacy, because they do not take into consideration unmeasurable new lesions [39-41].

- Immune-related RECIST (irRECIST): Developed in 2014 with the intention of aligning irRC with RECIST 1.1, irRECIST simplified immunotherapy response assessment and allowed comparison with other clinical trials that used RECIST criteria. According to the irRECIST criteria, new measurable lesions are added to baseline lesions in a concept called "total tumor burden measured". Similar to the irRC criteria, every disease progression case should be confirmed radiologically within a 4-week period [42, 43].

- Immune RECIST (iRECIST): These criteria were derived from a consensus among the RECIST work group, pharmaceutical industries, and the main regulatory agencies to standardize and validate immunotherapy response criteria. The iRECIST would become a consensus guideline assuring consistent data design and collection that would facilitate the analysis and compared interpretation of immunotherapy-based clinical trials. Unlike the other criteria, new lesions were not incorporated into baseline lesions, and thus, they were registered separately. One of the key concepts of iRECIST is immune-unconfirmed PD (iUPD), a category including cases at the first sign of PD to be confirmed within the next 4-8 weeks [41, 44, 45].

Immune-modified RECIST (imRECIST). imRECIST was described in 2018 and was inspired by the irRC criteria principles. New measurable lesions were incorporated into baseline target lesions (unlike in iRECIST). The imRECIST criteria relate patterns of response or progression to overall survival through indirect assessment criteria (such as progression-free survival) [46].

\section{Key points and recommendations}

- Although the classic RECIST criteria remain a useful tool to assess response to immunotherapy in the clini- cal practice, sometimes they underestimate the benefit of immunotherapy. Specific immune-related response criteria must be considered to evaluate patients under treatment with immunotherapy.

\section{Metabolic response criteria}

Given the limitations of conventional imaging techniques for the assessment of immunotherapy-treated patients, new functional and molecular imaging techniques were considered.

Metabolic response criteria for solid tumors were proposed, because tumor response to new targeted molecular therapies in the field of oncology might manifest as decreased 18F-FDG uptake without a marked reduction in tumor size (Table 3).

- European Organization for Research and Treatment of Cancer (EORTC): The first PET-based criteria were proposed in 1999 to assess metabolic response in solid tumors [47] and were the first criteria applied to assess metabolic response to immunotherapy [48]. A CR is defined as a resolution of FDG uptake within the tumor that is indistinguishable from the surrounding normal tissue. $\mathrm{A}>25 \%$ increase in tumor standard uptake value (SUV) or the appearance of new ${ }^{18}$ F-FDG-avid lesions defines progression.

- PET Response Criteria in Solid Tumors (PERCIST): Wahl et al. [49] proposed new response criteria applying the average SUV corrected by lean body mass (SUL) within a $1-\mathrm{cm}^{3}$ spherical volume of interest (SULpeak) [50]. More than $30 \%$ or 0.8 -unit increases in SULpeak or new ${ }^{18} \mathrm{~F}$-FDG-avid lesions indicated progression.CR was defined as ${ }^{18} \mathrm{~F}$-FDG uptake of target lesions lower than that of the liver and indistinguishable from the background.

- PET/CT Criteria for Early Prediction of Response to Checkpoint Inhibitor Therapy (PECRIT): Choet et al. [51] analyzed different criteria in 20 advanced melanoma patients treated with checkpoint inhibitors. They defined new morpho-functional early response criteria combining the change in the sum of RECIST 1.1-based target lesion diameters and the PERCIST SULpeak change in the hottest lesion, with a $95 \%$ diagnostic accuracy (sensitivity $100 \%$, specificity $93 \%$ ). A very interesting aspect of these criteria is the capacity to predict clinical benefit at 4 months, defined as RECIST CR, PR or SD based on an increase $>15.5 \%$ in the SULpeak of the hottest lesion. Therefore, the combination of morphologic and metabolic information constitutes the main tool for immunotherapy response assessment.

- PET Response Evaluation Criteria for Immunotherapy (PERCIMT). A prospective study was conducted recently 


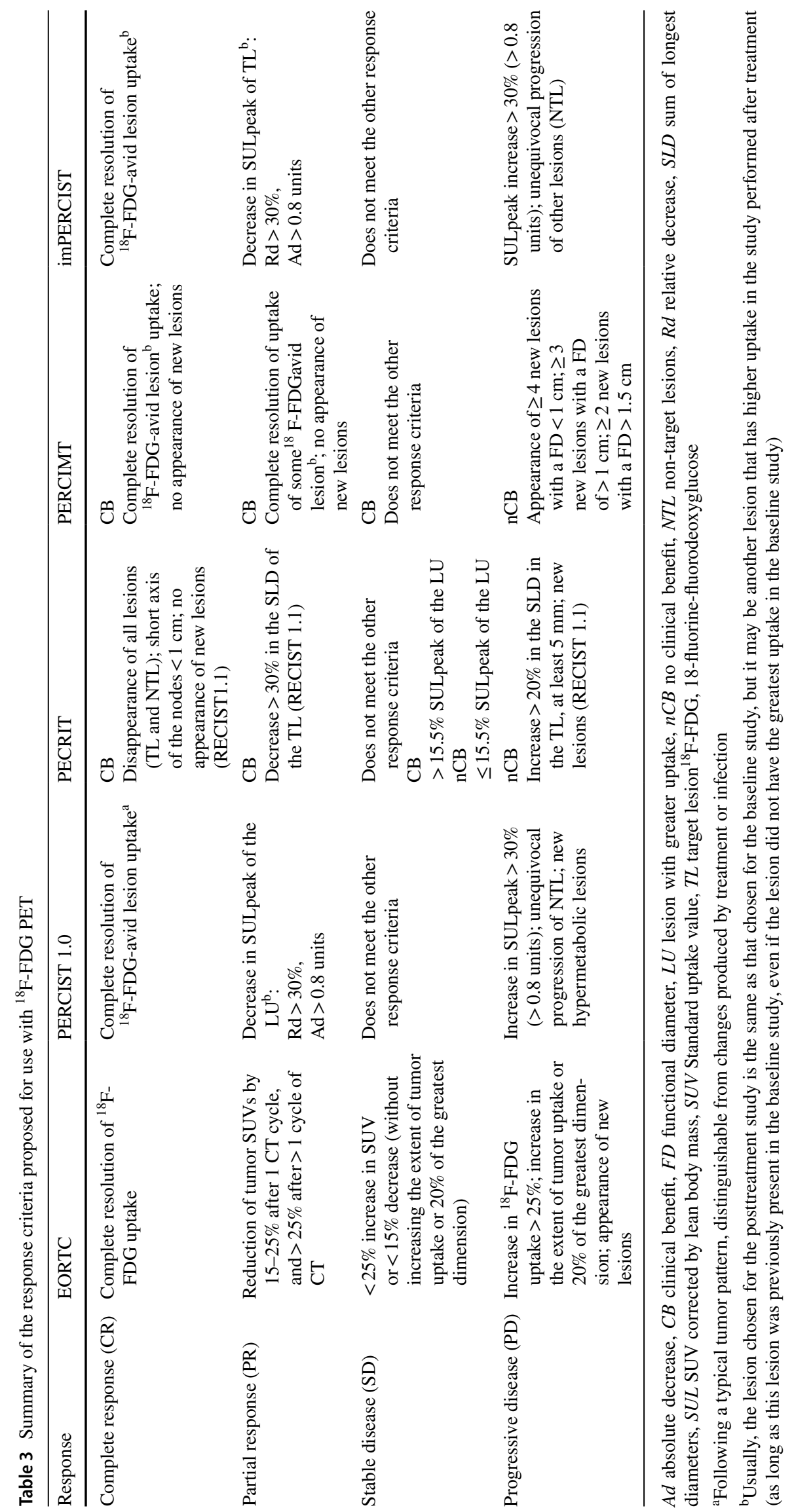


and included 41 metastatic melanoma patients treated with ipilimumab [52]. The authors defined clinical benefit as SD, PR and CR, and to predict outcome, they established optimal cut-off points for the number of new lesions according to their functional diameter measured in fused ${ }^{18}$ F-FDG PET/CT images. Neither maximum SUV (SUVmax) nor mean (SUVmean) changes during immunotherapy were correlated with clinical response, but they observed that a cut-off of four new lesions was predictive of treatment failure.

- Immunotherapy-modified PERCIST (imPERCIST): Described in 2019, these criteria do not necessarily consider new lesions as disease progression, as opposed to the PERCIST criteria. New lesions are included in ${ }^{18} \mathrm{~F}$ FDG tumor uptake quantification, and progression is defined as an increase $>30 \%$ in the sum of the SULpeak of up to 5 lesions from baseline and follow-up PET with a maximum of 2 lesions per organ [53]. In this setting, Ito et al. [53] analyzed the relationship between tumor ${ }^{18} \mathrm{~F}$ FDG uptake changes and survival in melanoma patients treated with ipilimumab according to both the PERCIST standard criteria and the new imPERCIST criteria. The authors concluded that PERCIST-based tumor response criteria correlated significantly with overall survival, and the new imPERCIST criteria improved this correlation as well as the prognostic value of ${ }^{18} \mathrm{~F}$-FDG PET/CTcompared to the results obtained using PERCIST.

\section{Key points and recommendations}

- FDG PET should be performed before immunotherapy (for evaluating the whole metabolic tumour burden), after 2-3 cycles of immunotherapy, and at the end of treatment (for response assessment). New PET-based criteria must be introduced in clinical practice. These criteria should be used instead of PET Response Criteria in Solid Tumors (PERCIST), which do not enable progression disease to be distinguished from the atypical response patterns to immunotherapy. imPERCIST criteria may improve the prediction of overall survival and the assessment of response in patients treated with immunotherapy, and PERCIMT or PECRIT may identify patients who achieve clinical benefit from immune treatment, defined as complete response, partial response or stable disease.

\section{Predictive and prognostic biomarkers}

One of the main clinical concerns related to immunotherapy is the identification of biomarkers allowing for the selection of patients who would respond to the therapy or for the prediction of atypical responses such as pseudoprogression or hyperprogression. Different types of biomarkers have been proposed, including PD-L1 expression, microsatellite instability, tumor mutational burden, $\mathrm{T}$ lymphocyte tumor infiltration or imaging tests.

\section{Serum/tissue biomarkers}

Immune checkpoint inhibitors constitute the main immunotherapy treatment in clinical practice. Some biomarkers have been proposed allowing the identification of patient groups who would benefit more from PD-1/PD-L1 inhibitors than from other therapeutic options. Without a doubt, the best-known biomarker is PD-L1 expression in tumor cells [54] or in immunologic system cells [55]. The usefulness of this marker is low in general, because we frequently observe patients who do not express PD-L1 and nevertheless respond to the treatment, and vice versa, and even in some studies, a greater response has been described in patients with lowPD-L1-expression tumor cells $[56,57]$. However, more than $50 \%$ PD-L1 tumor cell expression in non small cell lung cancer patients predicts higher effectiveness of pembrolizumab as a first-line treatment compared to the effectiveness of chemotherapy. Furthermore, PD-L1 expression is required by regulatory agencies like EMA to prescribe atezolizumab (PD-L1 $>5 \%$ ) or pembrolizumab (PD-L1 $>10 \%$ ) as a firstline treatment for urothelial cancer patients who are not candidates for chemotherapy with cisplatinum.

Nevertheless, the most relevant biomarker is microsatellite instability (observed in patients with DNA repair pathway alterations) and predicts a very high response rate to PD-1 /PDL-1 inhibitors [58]. This is why regulatory agencies have approved pembrolizumab for treating patients with tumors showing microsatellite instability, independent of their histological type. Finally, high tumor mutational burden has been linked to the effectiveness of immunotherapy [59], but given the conflicting results among several studies this biomarker has not yet been implanted in the clinic nor authorized by regulatory agencies.

Regarding biomarkers for monitoring disease progression, we would like to note that the interleukin- 8 serum level directly correlates with tumor burden and that variations in interleukin-8 during treatment with PD-1/PD-L1 inhibitors are associated with clinical response [60]. This type of biomarker could be useful for determining treatment effectiveness in conjunction with imaging tests, especially in cases of difficult response assessment, such as pseudoprogression.

\section{Key points and recommendations}

- Treatment with PD-1 / PD-L1 blockade should be prescribed based on biomarker expression in the following clinical situations: 
Metastatic non-small cell lung cancer and metastatic bladder cancer: monotherapy with immune checkpoints inhibitors is restricted to patients with positive expression of PD-L1 tumor cells (TPS) or a composite positive score (CPS).

Pembroliuzmab is approved by the FDA for the treatment of adult and pediatric patients with unresectable or metastatic, microsatellite instability-high (MSI-H) or mismatch repair deficient (dMMR) solid tumors that have progressed following prior treatment and who have no satisfactory alternative treatment options, and for the treatment of unresectable or metastatic MSI-H or dMMR colorectal cancer that has progressed following treatment with chemotherapy.

\section{Radiological biomarkers}

Several radiological findings derived from conventional imaging techniques might predict or suggest a response to immunotherapy. Thus, regardless of the classic criterion of size reduction, the halo sign can identify treatment response in patients with pulmonary metastases of melanoma undergoing treatment with immunotherapy [61]. Lesion density changes in computerized tomography (CT) have been considered as response criteria for other types of therapies. In this setting, Gray et al. evaluated melanoma patients treated with interferon and antiangiogenic therapy based on the morphology, attenuation, size, and structure (MASS) criteria, showing that they could predict progression-free survival and overall survival. Finally, new contrast media, such as ultrasmall superparamagnetic ironoxide (USPIO), tend to accumulate in tumor-associated macrophages and might be used for assessing immune cell infiltration levels in the tumor to evaluate possible immunotherapy responses by magnetic resonance imaging (MRI) [62].

Artificial intelligence has improved the analysis of medical images [63]. Radiomics, a method that extracts multiple quantitative data points from medical images, would allow the development of imaging biomarkers. Some image findings have been correlated with PD-L1 or IDO1 expression [63] or with the degree of tumor-infiltrating lymphocytes [64-66]. Chen et al. [66], by combining clinical and radiomic data extracted from the texture and morphological analysis of magnetic resonance images with hepatic-specific contrasts, could predict lymphocytic tumor-infiltration levels in hepatocellular carcinomas, and they found that tumors with higher lymphocyte infiltration were more homogeneous. Several parameters obtained from radiomic analysis of $\mathrm{CT}$ images were also correlated with clinical evolution after anti-PD-1 or PD-L1 therapies in patients with lung cancer [65, 67-70] or head and neck cancer [71].

Radiomics analysis of tumor structure, texture and heterogeneity might facilitate the obtainment of more accurate information on tumor biology. Radiomics could also be useful for assessing atypical responses and toxicities associated with immunotherapy. In this way, Tunali et al. [72] developed clinical radiological models able to predict possible hyperprogression and define poorer survival rates in patients treated with immunotherapy, and Colen et al. [73] established a radiomics-based model to predict immunotherapyinduced pneumonitis.

Functional imaging techniques have rarely been used as immunotherapy biomarkers. Nevertheless, whole-body MRI with diffusion-weighted sequences has been used for assessing rituximab-treated lymphoma patients, evidencing that apparent diffusion coefficient changes (ADCs) may allow the differentiation of responding from nonresponding patients $[74,75]$ and confirm an early response.

\section{Metabolic and molecular imaging biomarkers}

PET imaging techniques offer qualified imaging biomarkers that can be used not only as predictors of therapy response but also as prognostic factors. The most frequently used markers are SUV and new quantitative indexes, such as metabolic tumor volume (MTV) and total lesion glycolysis (TLG). Bastiannet et al. [76] correlated SUV in melanomainfiltrated lymph nodes with prognosis in patients with stage III melanoma, showing a significantly higher 5-year rate with lower (41\%) than with higher (24\%) SUVmean. However, other authors could not confirm this correlation between glucose uptake in PET and survival.

MTV defines the tumor volume (in $\mathrm{cm} 3$ ) by pathologic 18F-FDG uptake, and TLG is calculated by multiplying MTV by SUVmean, which weights the volumetric burden and metabolic activity of tumors. These biomarkers have demonstrated prognostic value in different solid and hematologic neoplastic tumors. Ito et al. [77] evaluated a cohort of 142 consecutive melanoma patients treated with ipilimumab, and MTV was an independent prognostic factor for overall survival $(p=0.001)$. Thus, patients with higher MTV showed lower survival than patients with below-average MTV (10.8 months versus 26 months, respectively). On the other hand, the combination of metabolic biomarkers with clinical prognostic factors such as age, LDL and brain metastasis allowed patient stratification into significantly different overall survival ranges.

${ }^{18}$ F-FDG PET radiomic analysis of tumors might provide more accurate information on tumor biology. The most commonly used parameters are those expressing 18F-FDG tumor metabolic heterogeneity, which has been correlated with higher relapse rates and has shown prognostic value for different tumor types [78]. A retrospective study conducted with patients with metastatic melanoma showed great metabolic heterogeneity in the metastasis of each patient and 
among patients. A higher 18F-FDG uptake showed a relationship with the lactate dehydrogenase (LDH) values [79]. Finally, a recent study showed better clinical outcome in colon cancer patients with higher tumor sphericity and lower tumor volume and 18F-FDG heterogeneity on PET [80].

\section{Key points and recommendations}

- Apart of different molecular markers (PD-L1 expression, microsatellite instability, and mismatch repair deficiency), there are emerging imaging-based biomarkers to predict and evaluate response to immunotherapy and the risk of immune-related adverse events. However, its usefulness in the clinical practice is still limited.

\section{Functional and molecular imaging advances for immunotherapy response assessment}

In preclinical models, multiparametric MRI (including contrast T2-, ADC- and T1-weighted imaging) has been used in glioblastoma multiforme brain tumors for an early response assessment of CAR-T and natural killer (NK) cell therapy [81, 82]. Promising techniques such as 19F MRI might facilitate in vivo assessment of monocytes and macrophages. The clinical use of functional imaging techniques has been limited in the assessment of immunotherapy response. The value of MRI diffusion has been demonstrated in lymphoma patients [74, 75, 83, 84], and CT perfusion has been applied for response assessment of interferon 1-treated metastatic renal cancer and carcinoid tumors [85, 86].Functional imaging techniques might also facilitate the differentiation between tumors and inflammatory sites in glioblastoma patients treated with dendritic cell immunotherapy [87].

On the other hand, immunoPET with specific labeled antibodies has great potential when the therapy depends on tumor target expression in lesions and enables assessment of biomarker expression evolution over time. Most immunoPET studies are preclinical or in the framework of clinical trials. 68 Ga-Granzyme B PET quantification is a highly sensitive and specific early predictor of therapeutic efficacy of checkpoint inhibitor therapy [88]. In non-small cell lung cancer patients, immunoPET with $89 \mathrm{Zr}$-nivolumab enabled the noninvasive quantification of PD-1/ PD-L1 tumor expression, demonstrating PD-L1 tumor expression heterogeneity between metastases of the same patient and among different patients [89]. In addition, PD-1 tumor expression was observed with 18F-BMS-986192 in patients with low PD-L1 expression, as determined by immunohistochemistry, most likely due to tumor PD-L1 expression heterogeneity. Therefore, immunoPET might identify patients despite low PD-L1 expression in biopsies, assessing tumor microenvironment changes induced by treatment with predictive and prognostic value. In a study conducted with $89 \mathrm{Zr}$-atezolizumab in lung, bladder and breast cancer patients, PET uptake exhibited high predictive value concerning treatment response and a strong correlation with survival [90].

Despite the promising data obtained by functional and molecular imaging techniques, clinical studies are necessaryto confirm these preliminary findings.

\section{Key points and recommendations}

- In the future, immunoPET imaging, which offers new radioactive tracers that target key immune pathways and cellular immune responses, should be used to improve patient's stratification, to predict the efficacy of immunotherapy, and to assess tumor response.

- Functional imaging techniques, including quantitative parameters derived from diffusion-weighted or perfusion imaging, have demonstrated a limited role in the assessment of immunotherapy. Nowadays, their clinical use would not be recommended beyond the research field.

\section{Conclusions}

Cancer immunotherapy has led to a significant breakthrough in patients' outcomes. In this setting, both conventional imaging techniques and classic response criteria have shown limited applicability for the assessment of immunotherapy response. To overcome these limitations, there is a growing need to maximize our ability to image the biological features associated with the interactions between tumors and the immune system. The combination of morphological and functional and/or metabolic response criteria together with the emergence of radiomics offers new approaches for the development of prognostic and predictive imaging biomarkers for immunotherapy response assessment.

Acknowledgements The authors would like to thank to Faustino Fernández Inclán (INCLAN CONGRESOS, S.L., Madrid, Spain) for the translation of a Spanish draft of the manuscript, and to Fernando Rico-Villademoros (COCIENTE SL, Madrid, Spain) for editorial assistance in the preparation of this manuscript. Their participation have been funded by the participating scientific societies.

Author contributions L. Leon-Mateos, MJ Garcia-Velloso, R GarcíaFigueiras have coordinated this manuscript and have contributed equally to its preparation. The remaining authors also contributed to the design, writing, and critical revision of the manuscript. All authors approved the final version to be published before it was submitted.

Funding Spanish Society of Medical Oncology (SEOM), the Spanish Society of Medical Radiology (SERAM), and Spanish Society of Nuclear Medicine and Molecular Imaging (SEMNIM) equally funded this consensus. 


\section{Compliance with ethical standards}

Conflict of interest LL-M has played a consultant or advisory role for Pfizer, Boehringer, Novartis, Roche, Astra Zeneca MSD, Sanofi; has received speaking honoraria from Novartis, Astra Zeneca, Bristol; Jansen, Pfizer, Astellas, Ipsen; and has received travel Grants from MSD, Ipsen. MJG-V declares relationships with the following companies: Amgen, Janssen, Takeda and Siemens Healthcare have invited MJGV to training courses. RG-F reports no conflict of interest. JFR$\mathrm{M}$ has been member of the advisory boards and consultant for BMS, Amgen, Novartis, Rainier; has received speaker honoraria from Roche, BMS, Novartis, MSD, Jansen, Pfizer, Astra-Zeneca; has received travel accommodations expenses from Astellas, Novartis, Roche, BMS, Pfizer, MSD; has received corporate-sponsored research grants from Astra-Zeneca, BMS, Amgen, Roche, Novartis, MSD, Jansen, Pfizer, Astellas, GSK, PharmaMar, Ipsen, Tesaro, Abbvie, Aprea Therapeutics, Eisai, Bayer, Merck. JLV-C has acted as advisory board member for AAA. MS has received speaker honorarium from Roche, BristolMyers Squibb, Boehringer Ingelheim; and has received research grants to institution from Roche. JLPG has received research grants and support from Roche, BMS, MSD, Ipsen, Eisai, Incyte, Janssen; has been member of the speakers' bureau and advisory boards of Roche, BMS, Ipsen, Eisai, MSD, Seattle Genetics; and has received travel support from Roche, MSD, BMS. MS-P reports no conflict of interests. LG reports no conflict of interest.

Ethical approval The manuscript does not contain any studies with human participants or animals performed by any of the authors.

Informed consent Informed Consent is not required for this type of project.

Open Access This article is licensed under a Creative Commons Attribution 4.0 International License, which permits use, sharing, adaptation, distribution and reproduction in any medium or format, as long as you give appropriate credit to the original author(s) and the source, provide a link to the Creative Commons licence, and indicate if changes were made. The images or other third party material in this article are included in the article's Creative Commons licence, unless indicated otherwise in a credit line to the material. If material is not included in the article's Creative Commons licence and your intended use is not permitted by statutory regulation or exceeds the permitted use, you will need to obtain permission directly from the copyright holder. To view a copy of this licence, visit http://creativecommons.org/licenses/by/4.0/.

\section{References}

1. Robert C, Long GV, Brady B, Dutriaux C, Maio M, Mortier $\mathrm{L}$, et al. Nivolumab in previously untreated melanoma without BRAF mutation. N Engl J Med. 2015;372:320-30.

2. Robert C, Schachter J, Long GV, Arance A, Grob JJ, Mortier L, et al. Pembrolizumab versus ipilimumab in advanced melanoma. N Engl J Med. 2015;372:2521-32.

3. Eggermont AM, Chiarion-Sileni V, Grob JJ, Dummer R, Wolchok JD, Schmidt H, et al. Prolonged survival in stage III melanoma with ipilimumab adjuvant therapy. N Engl J Med. 2016;375:1845-55.

4. Gandhi L, Rodriguez-Abreu D, Gadgeel S, Esteban E, Felip E, De Angelis F, et al. Pembrolizumab plus chemotherapy in metastatic non-small-cell lung cancer. N Engl J Med. 2018;378:2078-92.
5. Motzer RJ, Tannir NM, McDermott DF, Frontera OA, Melichar B, Choueiri TK, et al. Nivolumab plus ipilimumab versus sunitinib in advanced renal-cell carcinoma. N Engl J Med. 2018;378:1277-90.

6. Socinski MA, Jotte RM, Cappuzzo F, Orlandi F, Stroyakovskiy $\mathrm{D}$, Nogami N, et al. Atezolizumab for first-line treatment of metastatic nonsquamous NSCLC. N Engl J Med. 2018;378:2288-301.

7. Havel JJ, Chowell D, Chan TA. The evolving landscape of biomarkers for checkpoint inhibitor immunotherapy. Nat Rev Cancer. 2019;19:133-50.

8. Murphy K, Weaver C. Janeway's immunobiology. 9th ed. New York: Garland Science, Taylor \& Francis Group; 2017.

9. Atkins MB, Lotze MT, Dutcher JP, Fisher RI, Weiss G, Margolin $\mathrm{K}$, et al. High-dose recombinant interleukin 2 therapy for patients with metastatic melanoma: analysis of 270 patients treated between 1985 and 1993. J Clin Oncol. 1999;17:2105-16.

10. Redelman-Sidi G, Glickman MS, Bochner BH. The mechanism of action of BCG therapy for bladder cancer-a current perspective. Nat Rev Urol. 2014;11:153-62.

11. Rosenberg SA, Yang JC, Sherry RM, Kammula US, Hughes MS, Phan GQ, et al. Durable complete responses in heavily pretreated patients with metastatic melanoma using T-cell transfer immunotherapy. Clin Cancer Res. 2011;17:4550-7.

12. Grupp SA, Kalos M, Barrett D, Aplenc R, Porter DL, Rheingold $\mathrm{SR}$, et al. Chimeric antigen receptor-modified T cells for acute lymphoid leukemia. N Engl J Med. 2013;368:1509-18.

13. Andtbacka RH, Kaufman HL, Collichio F, Amatruda T, Senzer $\mathrm{N}$, Chesney J, et al. Talimogene laherparepvec improves durable response rate in patients with advanced melanoma. J Clin Oncol. 2015;33:2780-8.

14. McDermott DF, Drake CG, Sznol M, Choueiri TK, Powderly JD, Smith DC, et al. Survival, durable response, and long-term safety in patients with previously treated advanced renal cell carcinoma receiving nivolumab. J Clin Oncol. 2015;33:2013-20.

15. Schadendorf D, Hodi FS, Robert C, Weber JS, Margolin K, Hamid $\mathrm{O}$, et al. Pooled analysis of long-term survival data from phase II and phase III trials of ipilimumab in unresectable or metastatic melanoma. J Clin Oncol. 2015;33:1889-944.

16. Gettinger S, Horn L, Jackman D, Spigel D, Antonia S, Hellmann $\mathrm{M}$, et al. Five-year follow-up of nivolumab in previously treated advanced non-small-cell lung cancer: results from the CA209-003 study. J Clin Oncol. 2018;36:1675-84.

17. Di Giacomo AM, Danielli R, Guidoboni M, Calabro L, Carlucci D, Miracco C, et al. Therapeutic efficacy of ipilimumab, an anti-CTLA-4 monoclonal antibody, in patients with metastatic melanoma unresponsive to prior systemic treatments: clinical and immunological evidence from three patient cases. Cancer Immunol Immunother. 2009;58:1297-306.

18. Chiou VL, Burotto M. Pseudoprogression and immune-related response in solid tumors. J Clin Oncol. 2015;33:3541-3.

19. Wolchok JD, Hoos A, O'Day S, Weber JS, Hamid O, Lebbe C, et al. Guidelines for the evaluation of immune therapy activity in solid tumors: immune-related response criteria. Clin Cancer Res. 2009;15:7412-20.

20. Hodi FS, Hwu WJ, Kefford R, Weber JS, Daud A, Hamid O et al. Evaluation of Immune-Related Response Criteria and RECIST v1.1 in Patients With Advanced Melanoma Treated With Pembrolizumab. J Clin Oncol. 2016;34:1510-7.

21. Tazdait M, Mezquita L, Lahmar J, Ferrara R, Bidault F, Ammari $\mathrm{S}$ et al. Patterns of responses in metastatic NSCLC during PD- 1 or PDL-1 inhibitor therapy: Comparison of RECIST 1.1, irRECIST and iRECIST criteria. Eur J Cancer. 2018;88:38-47.

22. Solinas C, Porcu M, Hlavata Z, De Silva P, Puzzoni M, WillardGallo K, et al. Critical features and challenges associated with imaging in patients undergoing cancer immunotherapy. Crit Rev Oncol Hematol. 2017;120:13-211. 
23. Champiat S, Dercle L, Ammari S, Massard C, Hollebecque A, Postel-Vinay S, et al. Hyperprogressive disease is a new pattern of progression in cancer patients treated by Anti-PD-1/PD-L1. Clin Cancer Res. 2017;23:1920-8.

24. Borcoman E, Kanjanapan Y, Champiat S, Kato S, Servois V, Kurzrock R, et al. Novel patterns of response under immunotherapy. Ann Oncol. 2019;30:385-96.

25. Fuentes-Antras J, Provencio M, Diaz-Rubio E. Hyperprogression as a distinct outcome after immunotherapy. Cancer Treat Rev. 2018;70:16-211

26. Saada-Bouzid E, Defaucheux C, Karabajakian A, Coloma VP, Servois V, Paoletti X, et al. Hyperprogression during anti-PD-1/ PD-L1 therapy in patients with recurrent and/or metastatic head and neck squamous cell carcinoma. Ann Oncol. 2017;28:1605-11.

27. Ferrara R, Mezquita L, Texier M, Lahmar J, Audigier-Valette C, Tessonnier L, et al. Hyperprogressive disease in patients with advanced non-small cell lung cancer treated with PD-1/PD-L1 inhibitors or with single-agent chemotherapy. JAMA Oncol. 2018;4:1543-52.

28. Nishino M, Tirumani SH, Ramaiya NH, Hodi FS. Cancer immunotherapy and immune-related response assessment: The role of radiologists in the new arena of cancer treatment. Eur J Radiol. 2015;84:1259-68.

29. Naidoo J, Wang X, Woo KM, Iyriboz T, Halpenny D, Cunningham J, et al. Pneumonitis in patients treated with anti-programmed death-1/programmed death ligand 1 therapy. J Clin Oncol. 2017;35:709-17

30. Nishino M, Hatabu H, Sholl LM, Ramaiya NH. Thoracic complications of precision cancer therapies: a practical guide for radiologists in the new era of cancer care. Radiographics. 2017;37:1371-87.

31. Aide N, Hicks RJ, Le Tourneau C, Lheureux S, Fanti S, Lopci E. FDG PET/CT for assessing tumour response to immunotherapy: report on the EANM symposium on immune modulation and recent review of the literature. Eur J Nucl Med Mol Imaging. 2019;46:238-50

32. Prigent K, Aide N. ${ }^{18}$ F-Fludeoxyglucose PET/computed tomography for assessing tumor response to immunotherapy and detecting immune-related side effects: a checklist for the PET reader. PET Clin. 2020;15:1-10.

33. Gerwing M, Herrmann K, Helfen A, Schliemann C, Berdel WE, Eisenblatter $\mathrm{M}$, et al. The beginning of the end for conventional RECIST - novel therapies require novel imaging approaches. Nat Rev Clin Oncol. 2019;16:442-58.

34. Carter BW, Bhosale PR, Yang WT. Immunotherapy and the role of imaging. Cancer. 2018;124:2906-22.

35. Shanbhogue AK, Karnad AB, Prasad SR. Tumor response evaluation in oncology: current update. J Comput Assist Tomogr. 2010;34:479-84

36. Schwartz LH, Seymour L, Litiere S, Ford R, Gwyther S, Mandrekar $\mathrm{S}$ et al. RECIST 1.1 - Standardisation and disease-specific adaptations: Perspectives from the RECIST Working Group. Eur J Cancer. 2016;62:138-45.

37. Lalchandani UR, Sahai V, Hersberger K, Francis IR, Wasnik AP. A radiologist's guide to response evaluation criteria in solid tumors. Curr Probl Diagn Radiol. 2018;48:576-85.

38. Camidge DR, Doebele RC, Kerr KM. Comparing and contrasting predictive biomarkers for immunotherapy and targeted therapy of NSCLC. Nat Rev Clin Oncol. 2019;16:341-55.

39. Hoos A, Eggermont AM, Janetzki S, Hodi FS, Ibrahim R, Anderson A, et al. Improved endpoints for cancer immunotherapy trials. J Natl Cancer Inst. 2010;102:1388-97.

40. Pennock GK, Waterfield W, Wolchok JD. Patient responses to ipilimumab, a novel immunopotentiator for metastatic melanoma: how different are these from conventional treatment responses? Am J Clin Oncol. 2012;35:606-11.

41. Somarouthu B, Lee SI, Urban T, Sadow CA, Harris GJ, Kambadakone A. Immune-related tumour response assessment criteria: a comprehensive review. Br J Radiol. 2018;91:20170457.

42. Nishino M, Giobbie-Hurder A, Gargano M, Suda M, Ramaiya NH, Hodi FS. Developing a common language for tumor response to immunotherapy: immune-related response criteria using unidimensional measurements. Clin Cancer Res. 2013;19:3936-43.

43. Nishino M, Gargano M, Suda M, Ramaiya NH, Hodi FS. Optimizing immune-related tumor response assessment: does reducing the number of lesions impact response assessment in melanoma patients treated with ipilimumab? J Immunother Cancer. 2014;2:17

44. Seymour L, Bogaerts J, Perrone A, Ford R, Schwartz LH, Mandrekar S, et al. iRECIST: guidelines for response criteria for use in trials testing immunotherapeutics. Lancet Oncol. 2017;18:e143-e152152.

45. Inno A, Lo Russo G, Salgarello M, Corrao G, Casolino R, Galli $\mathrm{G}$, et al. The evolving landscape of criteria for evaluating tumor response in the era of cancer immunotherapy: From Karnofsky to iRECIST. Tumori. 2018;104:88-95.

46. Hodi FS, Ballinger M, Lyons B, Soria JC, Nishino M, Tabernero $\mathrm{J}$, et al. Immune-modified response evaluation criteria in solid tumors (imRECIST): refining guidelines to assess the clinical benefit of cancer immunotherapy. J Clin Oncol. 2018;36:850-8.

47. Young H, Baum R, Cremerius U, Herholz K, Hoekstra O, Lammertsma AA et al. Measurement of clinical and subclinical tumour response using $[18 \mathrm{~F}]$-fluorodeoxyglucose and positron emission tomography: review and 1999 EORTC recommendations. European Organization for Research and Treatment of Cancer (EORTC) PET Study Group. Eur J Cancer. 1999;35:1773-82.

48. Sachpekidis C, Larribere L, Pan L, Haberkorn U, Dimitrakopoulou-Strauss A, Hassel JC. Predictive value of early 18F-FDG PET/ $\mathrm{CT}$ studies for treatment response evaluation to ipilimumab in metastatic melanoma: preliminary results of an ongoing study. Eur J Nucl Med Mol Imaging. 2015;42:386-96.

49. Wahl RL, Jacene H, Kasamon Y, Lodge MA. From RECIST to PERCIST: Evolving Considerations for PET response criteria in solid tumors. J Nucl Med. 2009;50(Suppl 1):122S-S150150.

50. Tirkes T, Hollar MA, Tann M, Kohli MD, Akisik F, Sandrasegaran $\mathrm{K}$. Response criteria in oncologic imaging: review of traditional and new criteria. Radiographics. 2013;33:1323-41.

51. Cho SY, Lipson EJ, Im HJ, Rowe SP, Gonzalez EM, Blackford A, et al. Prediction of response to immune checkpoint inhibitor therapy using early-time-point (18)F-FDG PET/CT imaging in patients with advanced melanoma. J Nucl Med. 2017;58:1421-8.

52. Anwar H, Sachpekidis C, Winkler J, Kopp-Schneider A, Haberkorn U, Hassel JC, et al. Absolute number of new lesions on (18) F-FDG PET/CT is more predictive of clinical response than SUV changes in metastatic melanoma patients receiving ipilimumab. Eur J Nucl Med Mol Imaging. 2018;45:376-83.

53. Ito K, Teng R, Schoder H, Humm JL, Ni A, Michaud L, et al. (18) F-FDG PET/CT for monitoring of ipilimumab therapy in patients with metastatic melanoma. J Nucl Med. 2019;60:335-41.

54. Topalian SL, Hodi FS, Brahmer JR, Gettinger SN, Smith DC, McDermott DF, et al. Safety, activity, and immune correlates of anti-PD-1 antibody in cancer. N Engl J Med. 2012;366:2443-544.

55. Powles T, Eder JP, Fine GD, Braiteh FS, Loriot Y, Cruz C, et al. MPDL3280A (anti-PD-L1) treatment leads to clinical activity in metastatic bladder cancer. Nature. 2014;515:558-62.

56. Motzer RJ, Escudier B, McDermott DF, George S, Hammers HJ, Srinivas $\mathrm{S}$, et al. Nivolumab versus everolimus in advanced renalcell carcinoma. N Engl J Med. 2015;373:1803-13. 
57. Balar AV, Galsky MD, Rosenberg JE, Powles T, Petrylak DP, Bellmunt J, et al. Atezolizumab as first-line treatment in cisplatinineligible patients with locally advanced and metastatic urothelial carcinoma: a single-arm, multicentre, phase 2 trial. Lancet. 2017;389:67-766.

58. Le DT, Uram JN, Wang H, Bartlett BR, Kemberling H, Eyring $\mathrm{AD}$, et al. PD-1 blockade in tumors with mismatch-repair deficiency. N Engl J Med. 2015;372:2509-20.

59. Hellmann MD, Ciuleanu TE, Pluzanski A, Lee JS, Otterson GA, Audigier-Valette $\mathrm{C}$, et al. Nivolumab plus Ipilimumab in lung cancer with a high tumor mutational burden. N Engl J Med. 2018;378:2093-104.

60. Sanmamed MF, Perez-Gracia JL, Schalper KA, Fusco JP, Gonzalez A, Rodriguez-Ruiz ME, et al. Changes in serum interleukin-8 (IL-8) levels reflect and predict response to anti-PD-1 treatment in melanoma and non-small-cell lung cancer patients. Ann Oncol. 2017;28:1988-95.

61. Shrot S, Schachter J, Shapira-Frommer R, Besser MJ, Apter S. $\mathrm{CT}$ halo sign as an imaging marker for response to adoptive cell therapy in metastatic melanoma with pulmonary metastases. Eur Radiol. 2014;24:1251-6.

62. Iv M, Telischak N, Feng D, Holdsworth SJ, Yeom KW, DaldrupLink HE. Clinical applications of iron oxide nanoparticles for magnetic resonance imaging of brain tumors. Nanomedicine (Lond). 2015;10:993-1018.

63. Bi WL, Hosny A, Schabath MB, Giger ML, Birkbak NJ, Mehrtash A, et al. Artificial intelligence in cancer imaging: Clinical challenges and applications. CA Cancer J Clin. 2019;69:127-57.

64. Orooji M, Rakshit S, Beig N, Madabhushi A, Velcheti V. Computerized textural analysis of lung CT to enable quantification of tumor infiltrating lymphocytes in NSCLC. J Clin Oncol. 2016;34:11584.

65. Sun R, Limkin EJ, Vakalopoulou M, Dercle L, Champiat S, Han $\mathrm{SR}$, et al. A radiomics approach to assess tumour-infiltrating CD8 cells and response to anti-PD-1 or anti-PD-L1 immunotherapy: an imaging biomarker, retrospective multicohort study. Lancet Oncol. 2018;19:1180-91.

66. Chen S, Feng S, Wei J, Liu F, Li B, Li X, et al. Pretreatment prediction of immunoscore in hepatocellular cancer: a radiomicsbased clinical model based on Gd-EOB-DTPA-enhanced MRI imaging. Eur Radiol. 2019;29:4177-87.

67. Prasanna P, Tiwari P, Madabhushi A. Co-occurrence of local anisotropic gradient orientations (CoLlAGe): a new radiomics descriptor. Sci Rep. 2016;6:37241.

68. Velcheti V, Alilou M, Khunger M, Thawani R, Madabhushi A. Changes in computer extracted features of vessel tortuosity on CT scans post-treatment in responders compared to non-responders for non-small cell lung cancer on immunotherapy. J Clin Oncol. 2017;35:11518.

69. Xie Y, Khunger M, Thawani R, Velcheti V, Madabhushi A. Evolution of radiomic features on serial CT scans as an imaging based biomarker for evaluating response in patients with non-small cell lung cancer treated with nivolumab. J Clin Oncol. 2017;35:e14534.

70. Lee G, Bak SH, Lee HY, Choi JY, Park H, Lee SH, et al. Measurement variability in treatment response determination for non-small cell lung cancer: improvements using radiomics. J Thorac Imaging. 2019;34:103-15.

71. Prawira A, Dufort P, Halankar J, Paravasthu DM, Hansen A, Spreafico A et al. Development of a predictive radiomics signature for response to immune checkpoint inhibitors (ICIs) in patients with recurrent or metastatic squamous cell carcinoma of the head and neck (RM-SCCHN). Ann Oncol. 2016;27:vi328-50.

72. Tunali I, Gray JE, Qi J, Abdalah M, Jeong DK, Guvenis A, et al. Novel clinical and radiomic predictors of rapid disease progression phenotypes among lung cancer patients treated with immunotherapy: An early report. Lung Cancer. 2019;129:75-9.

73. Colen RR, Fujii T, Bilen MA, Kotrotsou A, Abrol S, Hess KR, et al. Radiomics to predict immunotherapy-induced pneumonitis: proof of concept. Invest New Drugs. 2018;36:601-7.

74. De Paepe K, Bevernage C, De Keyzer F, Wolter P, Gheysens $\mathrm{O}$, Janssens A, et al. Whole-body diffusion-weighted magnetic resonance imaging at 3 Tesla for early assessment of treatment response in non-Hodgkin lymphoma: a pilot study. Cancer Imaging. 2013;13:53-62.

75. Mayerhoefer ME, Karanikas G, Kletter K, Kiesewetter B, Weber M, Rausch I, et al. Can interim 18F-FDG PET or diffusionweighted MRI predict end-of-treatment outcome in FDG-Avid MALT lymphoma after rituximab-based therapy?: a preliminary study in 15 patients. Clin Nucl Med. 2016;41:837-43.

76. Bastiaannet E, Hoekstra OS, de Jong JR, Brouwers AH, Suurmeijer AJ, Hoekstra HJ. Prognostic value of the standardized uptake value for (18)F-fluorodeoxyglucose in patients with stage IIIB melanoma. Eur J Nucl Med Mol Imaging. 2012;39:1592-8.

77. Ito K, Schoder H, Teng R, Humm JL, Ni A, Wolchok JD, et al. Prognostic value of baseline metabolic tumor volume measured on (18)F-fluorodeoxyglucose positron emission tomography/computed tomography in melanoma patients treated with ipilimumab therapy. Eur J Nucl Med Mol Imaging. 2019;46:930-9.

78. Bundschuh RA, Dinges J, Neumann L, Seyfried M, Zsoter N, Papp $\mathrm{L}$, et al. Textural parameters of tumor heterogeneity in (1)(8)F-FDG $\mathrm{PET} / \mathrm{CT}$ for therapy response assessment and prognosis in patients with locally advanced rectal cancer. J Nucl Med. 2014;55:891-7.

79. de Heer EC, Brouwers AH, Boellaard R, Sluiter WJ, Diercks $\mathrm{GFH}$, Hospers GAP, et al. Mapping heterogeneity in glucose uptake in metastatic melanoma using quantitative (18)F-FDG PET/CT analysis. EJNMMI Res. 2018;8:101.

80. van Helden EJ, Vacher YJL, van Wieringen WN, van Velden FHP, Verheul HMW, Hoekstra OS, et al. Radiomics analysis of pre-treatment [(18)F]FDG PET/CT for patients with metastatic colorectal cancer undergoing palliative systemic treatment. Eur J Nucl Med Mol Imaging. 2018;45:2307-17.

81. Lazovic J, Jensen MC, Ferkassian E, Aguilar B, Raubitschek A, Jacobs RE. Imaging immune response in vivo: cytolytic action of genetically altered $\mathrm{T}$ cells directed to glioblastoma multiforme. Clin Cancer Res. 2008;14:3832-9.

82. Rygh CB, Wang J, Thuen M, Navarro AG, Huuse EM, Thorsen F, et al. Dynamic contrast enhanced MRI detects early response to adoptive NK cellular immunotherapy targeting the NG2 proteoglycan in a rat model of glioblastoma. PLoS ONE. 2014;9:e108414.

83. Horger M, Claussen C, Kramer U, Fenchel M, Lichy M, Kaufmann S. Very early indicators of response to systemic therapy in lymphoma patients based on alterations in water diffusivitya preliminary experience in 20 patients undergoing whole-body diffusion-weighted imaging. Eur J Radiol. 2014;83:1655-64.

84. Mayerhoefer ME, Raderer M, Jaeger U, Staber P, Kiesewetter B, Senn D, et al. Ultra-early response assessment in lymphoma treatment: [(18)F]FDG PET/MR captures changes in glucose metabolism and cell density within the first 72 hours of treatment. Eur J Nucl Med Mol Imaging. 2018;45:931-40.

85. Ng CS, Charnsangavej C, Wei W, Yao JC. Perfusion CT findings in patients with metastatic carcinoid tumors undergoing bevacizumab and interferon therapy. AJR Am J Roentgenol. 2011;196:569-76.

86. Ng CS, Wang X, Faria SC, Lin E, Charnsangavej C, Tannir NM. Perfusion CT in patients with metastatic renal cell carcinoma treated with interferon. AJR Am J Roentgenol. 2010;194:166-71.

87. Vrabec M, Van Cauter S, Himmelreich U, Van Gool SW, Sunaert S, De Vleeschouwer $S$, et al. MR perfusion and diffusion imaging in the follow-up of recurrent glioblastoma treated with dendritic cell immunotherapy: a pilot study. Neuroradiology. 2011;53:721-31. 
88. Larimer BM, Bloch E, Nesti S, Austin EE, Wehrenberg-Klee E, Boland G, et al. The effectiveness of checkpoint inhibitor combinations and administration timing can be measured by granzyme B PET imaging. Clin Cancer Res. 2019;25:1196-205.

89. Niemeijer AN, Leung D, Huisman MC, Bahce I, Hoekstra OS, van Dongen G, et al. Whole body PD-1 and PD-L1 positron emission tomography in patients with non-small-cell lung cancer. Nat Commun. 2018;9:4664.

90. Bensch F, van der Veen EL, Lub-de Hooge MN, Jorritsma-Smit A, Boellaard R, Kok IC, et al. (89)Zr-atezolizumab imaging as a non-invasive approach to assess clinical response to PD-L1 blockade in cancer. Nat Med. 2018;24:1852-8.

Publisher's Note Springer Nature remains neutral with regard to jurisdictional claims in published maps and institutional affiliations. 